\title{
A study on the Preconditions of Space Program Validation of Healthcare Architecture for Application of BIM Technology
}

\author{
병원건축의 BIM적용을 위한 공간프로그램유효성평가의 전제조건에 관한 연구 \\ Seong Joonho, 성준호* | Kim, Khilchae 김길채**
}

\begin{abstract}
Purpose: The planning and design of hospital generally requires the participation and consultation of skilled experts since it has more complex space program than any other buildings. Therefore, the BIM systems for the planning of hospital have been tried continuously. The purpose of this study is to identify the precondition for space Program validation of healthcare architecture based on BIM, which is recently receiving wide attention. Method: For this study, United States, Australia and Finland's guidelines were analyzed among the description space program validation system in 14 overseas BIM Guidelines. And the propose precondition that can be applied to healthcare architecture from among these description of space program validation items, target, process etc for General building. Result: 1) spatial program validation is the following four evaluation phase. Step 1: Standard setting phase Step 2: BIM model accuracy assessment phase Step 3: space validation phase Step 4: Performance evaluation phase 2) The standards for the building elements at Standards Setting stage is considered to the standards for the architectural elements of General building. 3) Healthcare Architecture Area calculation method is considered to be reasonable that borrowing the area calculation standard of general architecture according to the UIA of international standards. However, Be proposed of measuring method that reflect the efficiency of the design process step-by-step area calculation method. The performance assessment indicators of reflect the Hospital uniqueness have to developed. And the research needs to be carried out continuously according to the purpose for healthcare architecture of feature-oriented. Implications: In this paper like to understanding that precondition of space program validation considering the BIM. As a result, understanding to condition about step of the evaluation, the evaluation standards. Is expected to keep the focus on the development of performance indicators that reflect the uniqueness of the hospital for the efficient evaluation of the Hospital building.
\end{abstract}

Keywords BIM, Validation, Precondition, Healthcare Architecture

주 제 어 $\mathrm{BIM}$, 공간유효성, 전제조건, 병원건축

\section{Introduction}

\subsection{Background and Objective}

급속하게 변화하는 의료 환경에 대응하는 의료시설의 계획 및 설계는 더욱더 복잡해지고 있다. 건축주와 거주자

* Master's Course, Dept. of Architectural Engineering, Chungwoon University(Primary author: 6889545@naver.com)

** Professor, Ph.D, Dept. of Architectural Engineering, Chungwoon University(Corresponding author: ski8579@ gmail.com)
의 요구는 정리되어 설계지침과 공간 프로그램으로 제공 되어 지고 이에 대한 검토 작업은 발주지침에 대한 충실한 반영의 결과로 이해되어 진다. 의료시설계획가와 설계자의 의사반영에 많은 노력을 들이고 있는 이때에 건축 IT기술 인 BIM적용은 매우 반가운 일로 받아 들여 지고 있다.

$\mathrm{BIM}$ 을 통한 여러 장점이 있지만 최근에는 “공간 유효성 평가(space validation)”가 주목받고 있다. 특히 병원건축 의 공간유효성평가는 설계단계에서 주어진 구체화된 공간 프로그램과의 검토와 성능의 검토에 매우 활용도가 높을 것으로 예상하고 있다. 
많은 BIM전문가들은 BIM적용 공간유효성검토에 있어서 기능이 중시여기는 시설들에 관심을 가지고 시도하고 있다. 특히 병원건축과 법원 등에 적용사례들이 해외에서 발표되 고 있다. 이러한 상황에서 병원건축에 $\mathrm{BIM}$ 적용가능성을 검 토하고 이를 활용하기 위한 전제조건은 무엇인가 논리적 진행이 요구된다.

따라서 본 연구에서는 최근의 건축정보 디지털매체인 $\mathrm{BIM}$ 적용을 고려한 병원건축의 생애주기 동안의 공간유효 성 및 성능 검토를 위한 전제조건을 파악하는데 그 목적을 둔다.

\subsection{Research Methodologies and Scope}

병원건축의 BIM적용 공간유효성 평가를 위한 전제조건 을 파악하기 위하여 해외의 BIM 가이드라인 사례를 조사 분석하고 이를 통해서 병원건축의 전제 조건을 정리하였다. 조사를 위한 해외의 $\mathrm{BIM}$ 가이드라인 14 개 중 공간 프로그 램 유효성 평가 체계를 기술하고 있는 미국, 호주 그리고 핀 란드의 3 개 가이드라인 사례를 선정하였다. 나머지 가이드 라인들은 BIM 적용에 대한 일반적인 프로세스 중심의 가이 드라인으로 연구대상에서 제외하였다. 사례 분석 대상은 다음 [Table 1]과 같다.

연구방법으로는 3 개의 사례에서 논하고 있는 공간 프로 그램 유효성 평가 항목, 대상, 프로세스 등 일반 건축물의 경우를 대상으로 제시하고 있는 자료를 병원건축 특성에 적용 할 수 있는 구체적인 전제조건을 제시한다.

$\mathrm{GSA}$ 의 경우, 건물 설계, 시공, 유지관리 임대 등을 관장 하는 공공 회사로서, 공간관리의 효율화에 중점을 두고 있 으며, 공간의 명칭, 산정기준 등에 대한 기준과 분류들이 체 계적으로 되어 있다.

[Table 1] Aborad case of space program validation

\begin{tabular}{|c|c|c|c|c|}
\hline $\begin{array}{l}\text { Countries/ } \\
\text { Institutions }\end{array}$ & Guideline & Evaluation & Execution Phase & Tool \\
\hline $\begin{array}{c}\text { USA } \\
\text { (General } \\
\text { Service } \\
\text { Administration) }\end{array}$ & $\begin{array}{l}\text { BIM Guide } \\
\text { Series02 }\end{array}$ & $\begin{array}{c}\text { Spatial } \\
\text { Program } \\
\text { Validation }\end{array}$ & $\begin{array}{l}\text { - Schematic } \\
\text { Design } \\
\text { - Design } \\
\text { Development } \\
\text { - Construction } \\
\text { Documents }\end{array}$ & $\begin{array}{l}\text { Solibri } \\
\text { Model } \\
\text { Checker }\end{array}$ \\
\hline $\begin{array}{l}\text { Australia } \\
\text { (Cooperative } \\
\text { Research } \\
\text { Centre for } \\
\text { Construction } \\
\text { Innovation) }\end{array}$ & $\begin{array}{l}\text { National } \\
\text { Guidelines } \\
\text { for Digital } \\
\text { Modeling }\end{array}$ & $\begin{array}{l}\text { Model } \\
\text { Quality }\end{array}$ & $\begin{array}{l}\text { - Pre Design } \\
\text { - Schematic } \\
\text { Design } \\
\text { - Developed } \\
\text { Design } \\
\text { - Contract } \\
\text { Document }\end{array}$ & $\begin{array}{l}\text { Solibri } \\
\text { Model } \\
\text { Checker }\end{array}$ \\
\hline $\begin{array}{l}\text { Finland } \\
\text { (Senate } \\
\text { Property) }\end{array}$ & \begin{tabular}{|c|} 
BIM \\
Requirement \\
Vol 06
\end{tabular} & $\begin{array}{c}\text { Quality } \\
\text { Assurance }\end{array}$ & $\begin{array}{l}\text { - Design of } \\
\text { Alternatives, } \\
\text { - Early Design, } \\
\text { - Detail Design }\end{array}$ & $\begin{array}{c}\text { Solibri } \\
\text { Model } \\
\text { Checker }\end{array}$ \\
\hline
\end{tabular}

$\mathrm{GSA}$ 와 유사한 핀란드 $\mathrm{SP}$ 는 대학교, 사무실, 연구소, 문 화 및 기타 유형의 건물을 대상으로 관리하고 있다. 이에 각 공간에 관한 명칭, 유형, 면적 측정에 대한 체계적인 기준은 언급하고 있지 않지만 공간 모델의 평가 항목 및 평가 절차 에 대해 구체적으로 언급하고 있다.

호주의 $\mathrm{CRC}$ 는 국가 연구 개발 및 실행을 담당하는 기관 이다. GSA와 달리 자산, 설계, 시공 및 시설관리에 보다 효 율적인 기술을 보급하기 위한 연구에 중점을 두고 있다. 따 라서 어느 특정 건물의 유형에 대한 구체적인 내용보다는 $\mathrm{BIM}$ 프로젝트 진행시 공간 프로그램 유효성 평가의 방향에 관한 적용 기준 및 지침에 대한 예를 제시하고 있다.

\section{Theoretical Study}

\subsection{Building Performance and Space Program}

건물은 주어진 조건(context)에 의해 외부의 자연 현상 이나 주변 환경으로부터 다양한 힘 (예를 들어, 화재, 풍압, 태양열, 습기, 지진력 등)을 받으며, 이에 대응하기 위해 건 물의 각 부분들은 고유의 특성 즉, 속성(properties) 가지 게 된다. 이때 외부의 힘과 이에 대한 건물의 반응 사이에 건물의 사용목적에 맞는 동적인 균형이 이루어 질 때 그 건 물의 성능이 달성되었다고 한다. ${ }^{1)}$

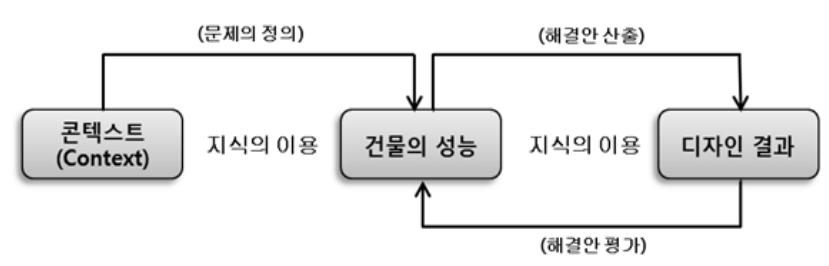

[Figure 1] The concept of performance-based design

\subsection{Design Process Focused on Performance}

성능 개념이 설계 방법에 본격적으로 도입되기 시작한 것은 1970년대 국제 건축 위원회 실무 위원회(CIB Working Commission) W60 "The Performance Concept in Building”이 구성되면서 부터이며 이 위원회는 1971년부터 14 차례 회의를 거쳐 보고서를 발간하였다. 이들 보고서에 서는 성능개념을 새롭게 정의하고 건축 생산 분야 전반에 적용 할 수 있는 실질적인 방법들을 제시하고 있다.

국내의 경우 "KS F 1010(건축물의 부위별 성능 분 류) 1976", “KS F 2273(조립용 판 및 그 구조 부분의 성능 시험 방법) $1977 ”$ 재정 및 1990년대부터 시작된 KS의 ISO 부합화 작업에 따라 국제적인 기준에 부합시키려는 노력이 시도되고 있으나, 아직 재료품질이나 공법 사양 등을 토대 로 이루어지고 있으며, 성능 중심의 설계 기법에 관한 연구

1) 전영일외 1인, 건축 디자인 이론, 기문당, 1997, p286 
는 국내 학계를 중심으로 시작되고 있는 단계에 있다. ${ }^{2)}$

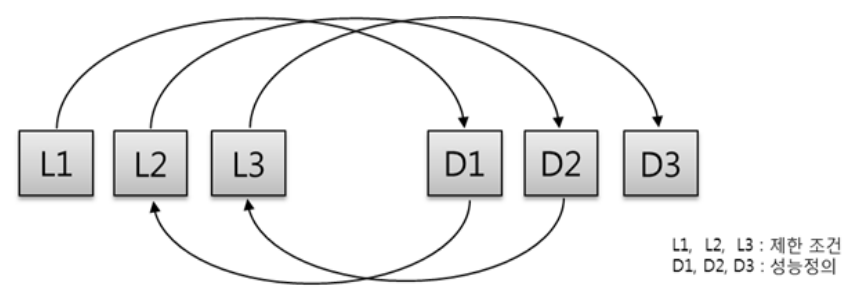

[Figure 2] Performance defined cycle(CIB Report Pub. 64, 1982)

\subsection{Space Program Validation}

건물의 설계 프로세스에서의 성능 개념이 처음으로 도 입된 것은 1960년대 후반으로서 마커스(Markus, 1969)와 B.P.R.U(1972)의 영향이 매우 크다. 마커스는 인간, 환경, 건물 생태 시스템의 개념적 모델을 제시하여 건물 성능의 개념을 정립 하였다. 그는 성능 평가 과정이 설계 대안산출 에 매우 중요하므로 설계 프로세스에 성능 평가 과정이 통 합 되어야한다고 주장하였다.

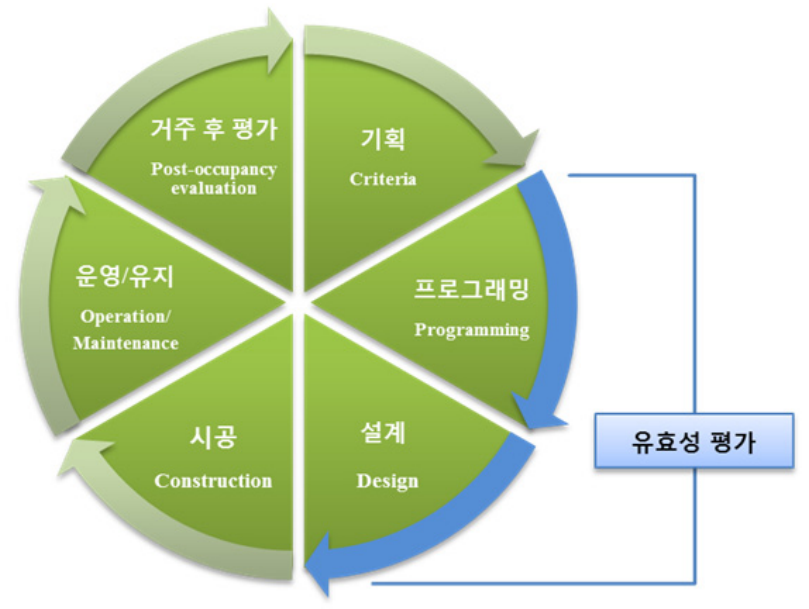

[Figure 3] 건물의 생애주기(Facility Standard for PBS, GSA, 2005 재편집)

건축물의 계획 평가에 대한 가장 보편적인 방법으로 거 주 후 평가(Post Occupancy Evaluation, 이하 POE)가 이 용되어 왔으며, 실제 건축물을 이용하는 거주자의 의견이 반영된다는 점에서 평가결과의 신뢰도와 활용도 측면에서 매우 긍정적으로 받아들여지고 있다. ${ }^{3)}$ 이러한 평가 결과 는 새로운 건축물 또는 기존 건축물의 개선을 위한 지침이 될 수 있으며, 유사한 건물의 유형과 비교할 수 있는 잣대로 서 활용 될 수 있다. 하지만 거주 후 평가는 계획안이 실제

2) 건설교통부, 한국건설교통기술평가원, 성능중심의 건설기준 개발 기본계획 수립 연구보고서, 2007 12, p4

3 ) 박영섭, 공간배치 평가모델 개발, 서울대학교 대학원 박사논문, 2009.04, p29
건축물로 구현된 이후에야 가능한 사후 평가에 해당되므로 계획안에는 그 결과를 반영할 수 없다는 한계를 가지고 있 다. 따라서 설계 단계에서의 적용 가능한 평가체계의 필요 성이 제기되고 있다. 공간 프로그램 유효성 평가는 이러한 설계 과정에서의 평가 체계에 주목한다.

\subsection{Previous Studies Related to Space Program Validation}

병원건축의 공간유효성 평가를 위한 국내연구는 $\mathrm{G} / \mathrm{N}($ 총면적/순면적)비의 개념을 적용하고 부문과 실들의 면적 을 데이터베이스화하는 연구(문창호, 1991)와 객체 지향 적 프로그래밍을 적용한 스페이스 검토방안을 연구(이낙 운, 1997)가 있다. 이들의 연구는 병원건축의 공간 프로그 램을 데이터베이스화와 전산화의 적용에 의미가 있는 연구 이다. 또한 병동부의 관계형 데이터베이스를 이용한 공간구 분, 평면분석이 가능한 전문가시스템개발에 관한 연구(김 길채, 2001)와 설계과정에서 즉각적인 공간유효성 검토가 가능한 연구(김길채, 2007)가 발표되었으며, 최근에는 BIM 을 적용한 병원건축의 공간유효성 평가를 위한 전문가 시 스템에 관한 연구(박영섭, 2010)가 있다. 그러나 공간 유효 성 평가를 위한 전제 조건 또는 기준에 대한 사항이 미흡하 여 분석결과 또는 평가 내용의 의미를 파악하기 곤란하다. 본 연구에서는 병원건축의 공간유효성 평가를 위한 전제되 어야 할 조건, 절차 또는 기준에 대한 기본적 연구에 초점을 두고 있다.

\section{Space Program Validation}

분석 사례들을 분석하여 여러 항목을 도출하고 이를 [Table 2]와 같이 건물 요소(벽체, 슬라브 등)와 공간(로 비, 화장실 등)으로 나누어 정리하였다.

그 결과 건물요소와 공간 모두 객체간의 중복, 충돌 및 간섭에 대한 사항과 객체의 속성과 기능의 유효성에 대하 여 검토하고 있음을 알 수 있었다. 또한 제공된 공간 프로그 램과의 공간 면적을 분석하는 공간유효성 평가와 공간 성 능(공간 효율성)에 대한 검토가 이루어지고 있다. 건축요소 와 공간은 모두 중복과 정확성 및 유효성 검토가 가능함을 알 수 있으며, 특히 공간의 합리적인 계획 및 설계를 평가할 수 있는 성능까지도 분석 가능함을 알 수 있다. 또한 이러한 과정을 몇 단계로 설정할 수 있다.

이를 정리하면 아래와 같이 결과 4단계의 유효성 평가 단계로 정리 할 수 있다.

1 단계 : 기준설정단계(건물요소와 공간에 대한 기준 설 정 등)

2단계 : BIM모델 정확성 평가 단계(설계된 BIM모델 평 가 등) 
3단계 : 공간 유효성 평가 단계(제공된 프로그램과 비교 평가 등)

4단계 : 성능 평가 단계(면적비, 에너지효율성, 효율성 및 경제성 평가 등)

\subsection{Validation Standard}

공간유효성 평가를 위해서는 사전에 평가기준설정단계 가 요구된다. 이를 위해서 본 연구에서는 건물요소와 공간 에 대한 기준을 사례조사를 통하여 분석하고 이를 병원건축 에 적용할 때 고려해야 할 전제조건에 대하여 정리하였다.
1) Building Element Standard

$\mathrm{BIM}$ 모델의 정확성 평가가 요구된다. 여기에는 객체 및 객체정보의 반영여부와 이들 객체간의 물리적 충돌과 누락 이 없도록 모델링과정의 가이드라인이 제공되어야 한다.

요구된 건물 요소의 포함은 공간 프로그램을 분석하기 위해 필요한 건물의 구성 요소를 말한다. $\mathrm{BIM}$ 저작 도구는 공간을 주변의 벽, 기둥, 창, 문 등과 같은 건물 요소로부터 정의 할 수 있고, 주변의 건물 요소 없이 공간 객체를 직접 정의 할 수 있다.

$\mathrm{GSA}$ 의 경우 요구되는 건물 요소의 벽, 기둥, 보, 슬래브,

[Table 2] Space Program Validation of case by case

\begin{tabular}{|c|c|c|c|c|}
\hline \multicolumn{2}{|c|}{ devide } & GSA & SP & $\mathrm{CRC}$ \\
\hline \multirow{5}{*}{ Elements } & Interference & Duplicate, and collisions & Duplicate, overlap, and collisions & Duplicate, overlap, and collisions \\
\hline & \multirow{4}{*}{$\begin{array}{l}\text { Accuracy } \\
\text { \& Validation }\end{array}$} & correct use of objects tool & correct use of objects tool & correct use of objects tool \\
\hline & & Hierarchically defined of Building elements & Defined Space Floor & Defined Space Floor \\
\hline & & N/A & Defined Building elements Type & N/A \\
\hline & & $\begin{array}{l}\text { Required Building elements(Wall, Opening, } \\
\text { Door, Window, Slab, Beam, Column) }\end{array}$ & Required Building elements & Required Building elements \\
\hline \multirow{28}{*}{ Space } & Interference & Duplicate, and overlap & overlap & overlap \\
\hline & \multirow{13}{*}{$\begin{array}{l}\text { Accuracy } \\
\text { \& Validation }\end{array}$} & $\begin{array}{l}\text { The gap between the space and the adjacent } \\
\text { boundary elements }\end{array}$ & $\begin{array}{l}\text { Whether to match the shape and } \\
\text { size of the wall and the space }\end{array}$ & N/A \\
\hline & & $\begin{array}{l}\text { The relationship between the space and the } \\
\text { space around the building components }\end{array}$ & N/A & N/A \\
\hline & & Defined Floor & Defined Floor & Defined Floor \\
\hline & & Minimum Area-0.9st $\left(0.85 \mathrm{~m}^{2}\right)$ & N/A & Minimum Area-1m² \\
\hline & & Defined Space Hight & Defined Space Hight & Space Hight more than $500 \mathrm{~mm}$ \\
\hline & & Defined Zone & Defined MEP Space & N/A \\
\hline & & $\begin{array}{l}\text { Compare space and area with Model and } \\
\text { Space program }\end{array}$ & $\begin{array}{l}\text { Compare space and area with } \\
\text { Model and Space program }\end{array}$ & $\begin{array}{l}\text { Compare space and area with } \\
\text { Model and Space program }\end{array}$ \\
\hline & & Include space name and number & Include space name and number & Include space name and number \\
\hline & & Defined user name of Space & Defined Space Unique ID & N/A \\
\hline & & GSA STAR space type & N/A & N/A \\
\hline & & Defined "Full_Floor Space" & N/A & N/A \\
\hline & & GSA Gross area & BIM model Gross area & N/A \\
\hline & & N/A & $\begin{array}{l}\text { Accuracy of the measurement of } \\
\text { the area. }\end{array}$ & N/A \\
\hline & \multirow{14}{*}{ Performance } & N/A & Net area ratio & N/A \\
\hline & & Major vertical penetrations & N/A & N/A \\
\hline & & Floor rentable area & N/A & N/A \\
\hline & & usable area & Net ratio & N/A \\
\hline & & office area & N/A & N/A \\
\hline & & Floor usable area & N/A & N/A \\
\hline & & Floor common area & N/A & N/A \\
\hline & & Basic Rentable Area & N/A & N/A \\
\hline & & Building Rentable Area & N/A & N/A \\
\hline & & Building R/U Ratio & N/A & N/A \\
\hline & & Rentable Area & N/A & N/A \\
\hline & & R/U Ratio & N/A & N/A \\
\hline & & N/A & N/A & $\begin{array}{l}\text { Code Checking of Evacuation } \\
\text { Route }\end{array}$ \\
\hline & & N/A & N/A & code checking of Accessibility \\
\hline
\end{tabular}


문, 창문에 대해 제시하고 있으며, $\mathrm{SP}$ 의 경우 공간 객체만 을 요구하거나 건물 요소와 함께 요구하는 두 가지 모두 사 용하고 있다. 이는 공간 유효성 평가의 설계 프로세스를 고 려한 결과이며, SP의 경우 Spatial BIM, Building Element $\mathrm{BIM}$ 의 설계 단계 별 생성되는 $\mathrm{BIM}$ 모델을 정의하고 이들로 부터 평가 체계를 구축하고 있다. $\mathrm{CRC}$ 의 경우 요구된 건물 구성 요소의 포함여부를 검토하고는 있지만 그 내용은 구 체적으로 제시하지 않고 있다. 이러한 건물 요소의 요구는 불필요한 객체의 사용을 자제하고 해당 공간과 그 주변의 건물 요소와의 관계를 통해 공간의 접근성을 분석 할 수 있 는 기반을 마련 할 수 있다.

건물 요소의 경우 공간을 정의하기 위한 주변 객체로서 정확한 모델링이 요구되며, 공간은 공간을 통해 산정되는 면적과 체적 등의 공간의 규모에 대한 후행 업무가 요구되 므로 모델링의 정확성이 요구된다. 조사된 해외 사례 모두 객체의 중복, 충돌, 간섭에 대한 검토 체계를 마련하고 있는 것은 정확한 정보 구축을 위한 선행 조건으로 해석 할 수 있을 것이다. 본 연구에서는 객체의 중복, 충돌 등에 검토사 항은 '간섭사항 검토'로 정의하며 조사된 사례의 내용은 다 음 [Table 3]과 같다.

분석한 사례들의 경우 일반 건축물의 모델링 기준이 설 정된 단계에서 건축부재의 간섭 및 오류에 대한 검토를 논 하고 있는 것으로 병원건축물 또한 일반 건축물의 건축요 소에 대한 기준을 따라야 할 것으로 생각되며, 이에 대한 기 준이 조속히 국내에서도 제공되어야 할 과제라고 사료된다.

[Table 3] Interference Check

\begin{tabular}{l}
\hline Space and Building Interference check \\
\hline - Duplicate, overlap, and collisions between building elements \\
\hline - Duplicate, overlap, and collisions between Space \\
\hline - Collisions Check between Space and Building elements \\
\hline
\end{tabular}

2) Space and Calculation Standard

건축요소에 의하여 형성된 공간은 건축행위의 궁극적인 목적이기도 하다. 이에 대한 유효성 평가를 위해서는 공간 의 정의와 구성 그리고 공간산정방식에 대한 기준이 명확 히 제공되어야 한다.

$\mathrm{BIM}$ 의 활용을 위해서는 모델 정보에 관한 사전 정의가 되어야 한다. 요구된 정보를 파악하여 사례의 공간 프로그 램 유효성 평가의 활용 방안을 예측할 수 있다. BIM 모델 요구정보 정의 및 검토에 대한 내용은 다음 [Table 4]와 같 다.

공간속성 정보의 경우 GSA가 국가별 공통된 항목을 가 장 많이 포함 하고 있으며 다음으로 $\mathrm{SP}, \mathrm{CRC}$ 의 순으로 나 타났다. 이는 품질관리의 측면에서 언급하는 $\mathrm{SP}$ 와 $\mathrm{CRC}$ 의 사례와 달리 공간 프로그램 유효성 평가에 대해 중점적으
로 다루고 있는 미국 GSA의 특성에 따른 결과로 볼 수 있으 며, 공간 프로그램 유효성 평가의 활용도가 가장 높은 것으 로 판단할 수 있다. 또한 세부 내용으로 공간 이름, 유형, 면 적, 높이, 고유 $\mathrm{ID}$, 층에 대한 정보와 공간을 정의하기 위한 최소 크기 기준 등은 공간 객체 하나의 포함 되어야 할 정 보이며, BIM 기술을 활용함으로서 공간 객체에 구축 할 수 있는 정보의 확장성을 보여준다.

[Table 4] Require Information of BIM Model

\begin{tabular}{|c|c|c|c|c|}
\hline \multicolumn{2}{|r|}{ Contents } & GSA & SP & CRC \\
\hline \multirow{10}{*}{ Attribute } & - Defined Space name & $\mathrm{O}$ & $\mathrm{O}$ & $\mathrm{O}$ \\
\hline & - Defined Space Type & $\mathrm{O}$ & $\mathrm{O}$ & 0 \\
\hline & - Defined Space Area & $\mathrm{O}$ & $\mathrm{O}$ & $\mathrm{O}$ \\
\hline & - Defined Space Hight & 0 & $\mathrm{O}$ & $\mathrm{O}$ \\
\hline & - Defined Space Unique ID & $\mathrm{O}$ & $\mathrm{O}$ & $\mathrm{O}$ \\
\hline & - Defined Space Floor & $\mathrm{O}$ & $\mathrm{O}$ & $\mathrm{O}$ \\
\hline & - Defined Space Number & $\mathrm{O}$ & $\mathrm{n} / \mathrm{a}$ & $\mathrm{O}$ \\
\hline & - Defined user name of Space & $\mathrm{O}$ & $\mathrm{n} / \mathrm{a}$ & $\mathrm{n} / \mathrm{a}$ \\
\hline & - Defined "Full_Floor Space" & $\mathrm{O}$ & $\mathrm{n} / \mathrm{a}$ & $\mathrm{n} / \mathrm{a}$ \\
\hline & - Defined MEP Space & $\mathrm{n} / \mathrm{a}$ & 0 & $\mathrm{n} / \mathrm{a}$ \\
\hline
\end{tabular}

유효성 평가는 제시된 공간 프로그램의 내용과 설계 안 이 반영된 BIM 모델과의 일치여부를 평가하는 것이다. 여 기에는 성능 기준 별 제시된 공간이름, 공간유형의 명칭에 대한 정의와 이들의 양적인 내용으로 면적, 높이, 수량 등의 항목을 포함하고 있다. SP와 CRC의 경우 이러한 공간 기준 에 대해 개념적으로 설명하고 있으며 GSA의 경우는 공간 의 이름, 유형, 면적 산정에 관한 기준을 마련하고 있다. 이 는 앞서 언급한 공간 성능 기준에 관한 지침의 활용여부와 관련이 있다. 즉, $\mathrm{GSA}$ 의 경우 오피스 건물에 관한 성능 기 준을 마련하고 이를 위한 공간 분류 및 면적 산정기준이 마 련되어 있어 이를 유효성 평가 기준으로서 활용 할 수 있는 데 반해 $\mathrm{SP}$ 와 $\mathrm{CRC}$ 의 경우 성능 기준에 대해 포괄적으로 접 근하고 있어 유효성 평가 기준은 구체적인 내용을 포함하 지 않고 있다.[Table 5] 따라서 유효성 평가 기준에 대해서 는 미국 GSA를 중점으로 분석하고자 한다. GSA는 성능 기 준에 따른 공간 분류 체계 및 면적 산정 기준을 제시하고 있다. GSA의 Public Building Service(이하 PBS)에서 PBS Business Assignment Guide ${ }^{4)}$ 를 제공하여 공간 명칭에 대 한 유효성 평가를 실시하고 있다.

$\mathrm{PBS}$ 는 공간의 이름, 유형, 카테고리로 분류하며 분류된 각 공간은 면적을 산정하여 관련 유형과 카테고리를 활용 한 건물의 총면적 아래로 임대 가능면적, 비 임대 면적으로

4) GSA PBS, National Business Space Assignment Policy, 2009년 5 월, pp.1-27 
분류하고 각각 세부 유형과 해당 공간을 분류 제시하고 있 다(Table 6).

[Table 5] Space classification and Area Measurement

\begin{tabular}{|c|c|c|c|}
\hline Division & GSA & $\mathrm{SP}$ & $\mathrm{CRC}$ \\
\hline $\begin{array}{l}\text { Define Room } \\
\text { name }\end{array}$ & 108 Room Name & $\begin{array}{l}\text { participants } \\
\text { discuss }\end{array}$ & $\begin{array}{l}\text { participants } \\
\text { discuss or } \\
\text { User Define }\end{array}$ \\
\hline $\begin{array}{l}\text { Define Space } \\
\text { Type }\end{array}$ & 35 Space type & $\begin{array}{l}\text { participants } \\
\text { discuss }\end{array}$ & $\begin{array}{l}\text { participants } \\
\text { discuss or } \\
\text { User Define }\end{array}$ \\
\hline $\begin{array}{l}\text { Area } \\
\text { Measurement }\end{array}$ & ANSI/BOMA & N/A & N/A \\
\hline $\begin{array}{l}\text { Define Space } \\
\text { High }\end{array}$ & $\begin{array}{l}\text { Floor finish to } \\
\text { ceiling finish }\end{array}$ & N/A & N/A \\
\hline $\begin{array}{l}\text { Minimum } \\
\text { Space Area }\end{array}$ & $0.9 \mathrm{sf}\left(0.85 \mathrm{~m}^{2}\right)$ & N/A & $\begin{array}{l}\text { More than } \\
1 \mathrm{~m}^{2} \text { (Area), } \\
500 \mathrm{~mm} \text { ( Hight) }\end{array}$ \\
\hline Unique ID & \multicolumn{3}{|c|}{ Supported by BIM authoring software } \\
\hline $\begin{array}{l}\text { Define Space } \\
\text { Floor }\end{array}$ & \multicolumn{3}{|c|}{$\begin{array}{l}\text { Defined and the corresponding layer of the floor } \\
\text { space, including information }\end{array}$} \\
\hline
\end{tabular}

[Table 6] Space classification system of the GSA

\begin{tabular}{|c|c|c|}
\hline Division & Contents & ex \\
\hline $\begin{array}{l}\text { Building } \\
\text { Area }\end{array}$ & $\begin{array}{l}\text { Gross area }=\text { Rentable Area }+ \\
\text { Non Assignable Area }\end{array}$ & $\begin{array}{l}\text { - Gross Area } \\
\text { - Rentable Area } \\
\text { - Non } \\
\text { Assignable Area }\end{array}$ \\
\hline $\begin{array}{l}\text { PBS \& ANSI } \\
\text { Category }\end{array}$ & $\begin{array}{l}\text { Included in } 5 \text { Category. Office } \\
\text { Area, Building Common, } \\
\text { Floor Common categories are } \\
\text { included in Rentable Area. } \\
\text { Vertical Penetration, PBS } \\
\text { Specific Area categories are } \\
\text { included in Non Assignable } \\
\text { Area. }\end{array}$ & $\begin{array}{l}\text { - Office Area } \\
\text { - Building } \\
\text { Common, } \\
\text { - Floor } \\
\text { Common, } \\
\text { - Vertical } \\
\text { Penetration } \\
\text { - PBS Specific } \\
\text { Area, }\end{array}$ \\
\hline $\begin{array}{l}\text { PBS Usable } \\
\text { Area }\end{array}$ & $\begin{array}{l}\text { Included in Assignable Space, } \\
\text { Joint Use Space, they are } \\
\text { associated with the ANSI/BOMA }\end{array}$ & $\begin{array}{l}\text { - Assignable } \\
\text { Space } \\
\text { - Joint Use } \\
\text { Space }\end{array}$ \\
\hline $\begin{array}{l}\text { PBS Space } \\
\text { Category }\end{array}$ & $\begin{array}{l}\text { Included in } 15 \text { Category and } \\
\text { associated with the ANSI/ } \\
\text { BOMA. }\end{array}$ & $\begin{array}{l}\text { - Facility } \\
\text { Common } \\
\text { - Building } \\
\text { Common } \\
\text { - Community } \\
\text { Joint Use }\end{array}$ \\
\hline $\begin{array}{l}\text { PBS(Space) } \\
\text { STAR } \\
\text { Space Type }\end{array}$ & $\begin{array}{l}\text { Included in } 35 \text { Type and they } \\
\text { are defined according to the } \\
\text { characteristics of the room. } \\
\text { Abbreviated to } 3 \text { letters. }\end{array}$ & $\begin{array}{l}\text { - AUD } \\
\text { - CRD } \\
-\mathrm{TTO} \\
-\mathrm{CRH} \\
-\mathrm{MCH}\end{array}$ \\
\hline Room Name & Defined 108 Room Name & $\begin{array}{l}\text { - ATRIUM } \\
\text { - WAREHOUSE } \\
\text { - OFFICE }\end{array}$ \\
\hline
\end{tabular}

분류된 내용의 최하위 개념인 실 이름은 총 108 개의 실 이름을 정의하고 있으며, 이를 총 38 가지의 PBS STAR Space Type으로 분류하고 있다. PBS STAR Space Type은 공간 유형을 식별하는 최하위 유형으로 실의 증축에 관한 정보로서 3글자 약어로 PBS 저장 시스템에 기록된다.

PBS Space Category는 15개의 카테고리로서 분류 되고 실의 사용 현황에 관한 정보로서 사용 된다. Space Type의 사용자에 따라 Space Category에서는 건물 공용(Building Common), 시설공용(Facility Common), 전용(Assigned), 커뮤니티 공용(Community joint use) 등으로 분류된다.

Space Usable Area는 임대면적 (OFFICE Area)안에서 전용 공간과(Assignable Space)과 공용 공간(Joint Use Space)으로 분류하며 이중 공용 공간은 건물전체 공용과 달리 순수 오피스 공간 사이에서 존재하는 공용 부분으로 서 예를 들어 내부 복도, 화장실 등을 의미한다.

PBS \& ANSI Category는 PBS와 ANSI의 공통된 분 류 체계로서 임대면적(OFFICE Area), 건물 공용(Building Common), 층 공용(Floor Common), 수직관통 영 역(Vertical Penetration), PBS 특수 면적(PBS Specific Area)으로 총 5가지로 분류하고 있다. 또한 이를 기준으로 면적 측정 기준을 달리 하며 각 상황별 면적 측정 방법을 제시하고 있다(Figure 4, 5).

$\mathrm{PBS}$ 의 공간 분류의 특징은 공간에 관하여 사용자의 관점 에서 전용여부에 따라 상황에 맞게 구분함으로써 임대면적 산출시 합리성을 제고하고 있다는 점이다. 예들 들어 공용 면적 복도의 경우 이는 1 차적으로 시설공용, 임대공용, 건 물 공용 중 복도가 지원하는 사용 공간에 따라 정의하고 이 는 건물 전체 공용면적과 층 공용면적으로 분류되고 점유 에 따라 면적 측정 기준을 달리 한다. 이는 공간의 사용여부 에 따라 세세한 분류 및 측정을 통해 결국 임대면적을 합리 적으로 산출하여 임대건물의 성격상 임차인과의 이해관계 를 보다 명확히 할 수 있을 것이다.

$\mathrm{GSA}$ 의 공간 프로그램 유효성 평가의 전반적인 목적은 GSA 공간 프로그램 요구 조건과 관련하여 설계 성능을 효 율적으로 정확하게 평가하여 궁극적으로는 평가의 자동화 와 구축된 공간 정보로부터 설계의 질을 높이고자 하는 것 이다. 따라서 위 분류 기준에 따라 구축된 공간 정보는 공간 데이터 관리팀(SDM)의 CAFM (Computer Aided Facilities Management)데이터 베이스에 기록되고 유사한 공간 유형 및 건물 유형을 비교하여 공간 효율성을 검토 한다. 즉, 공간에 관한 체계적인 분류를 통해 일관된 정보를 구축 하여 건물의 비교가 용이해지고 보다 신뢰성 있는 정보를 얻을 수 있어 건물의 품질을 높이는 것으로 사료된다. GSA 에서 활용하는 PBS 공간 분류 체계의 개념도는 다음 [Figure 6]과 같다. 

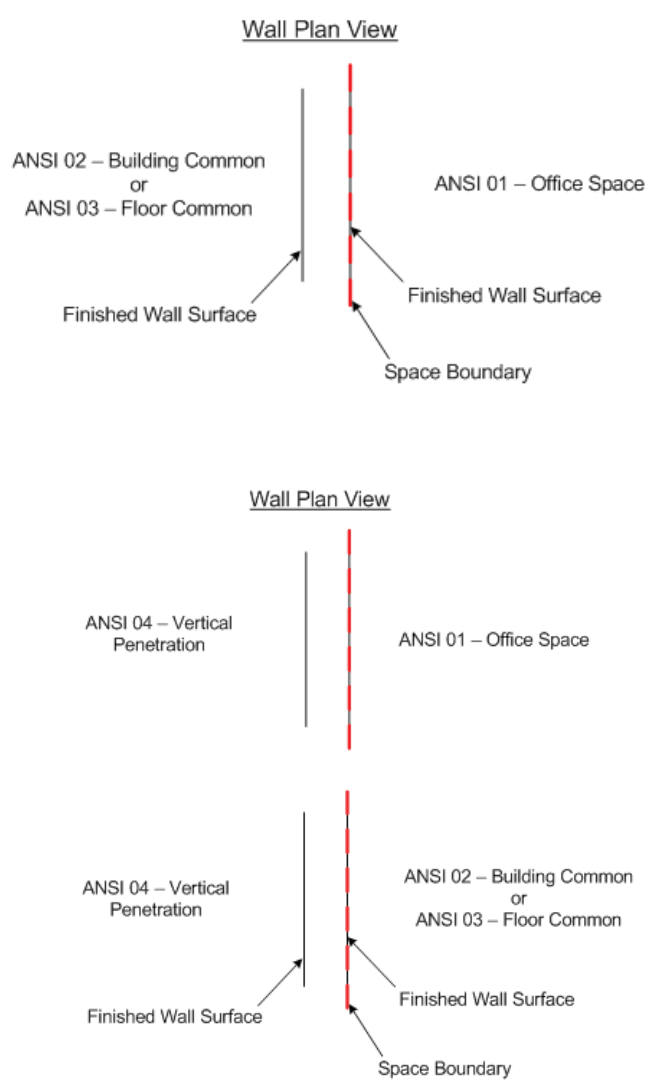

[Figure 4] PBS \& ANSI Category is different (ANSI $04>03>02$ $>01$ Discerning Dimensions)

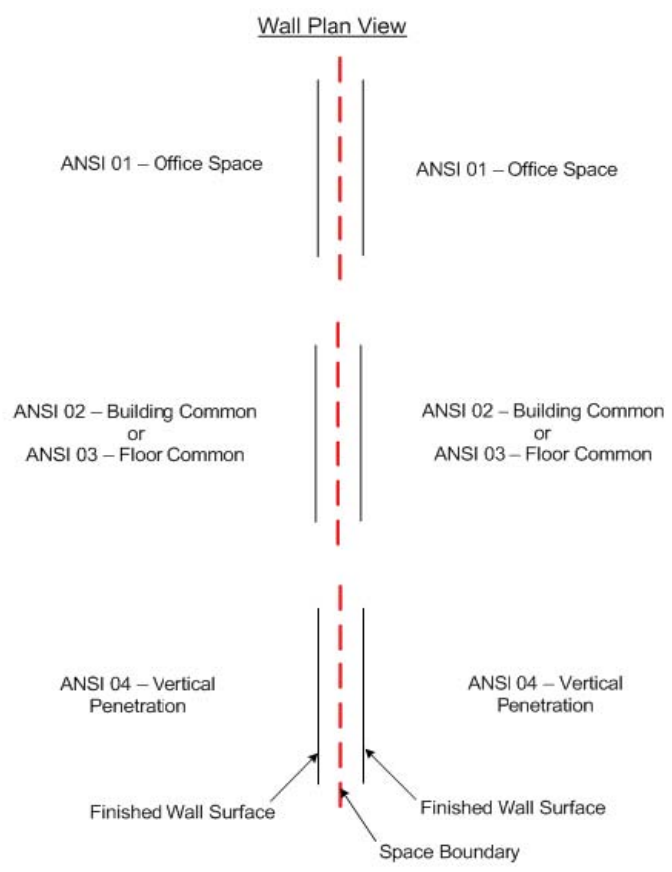

[Figure 5] PBS \& ANSI Category is the same(Centerline of the wall)

병원건축에서는 면적산정기준을 국제적인 기준인 UIA를 따라 일반건축물의 면적산정기준 차용하여 산정하는 것이 타당한 것으로 판단된다. 다만, 설계프로세스 단계별 면적
산정방법을 설계효율성을 반영하는 방안을 적용하는 것을 제안한다.

병원건축의 공간의 정의와 체계는 미국 $\mathrm{GSA}$ 의 경우와 같이 공간 즉 단위 실들의 명칭과 그에 따른 속성(기능 및 유형 등)을 정의하여야 하며, 각 단위 실들의 체계를 구축 하여야 할 것으로 판단된다. 기존 연구에서 병원의 부문구 성을 체계화하여 기준을 설정하여야 할 필요가 있다.

\subsection{Space Validation}

해외 사례 모두 공간 유효성 평가는 제공된 공간 프로그 램을 기준으로 BIM 모델의 반영 여부를 평가 하고 있다. 그 러나 구체적인 프로그램의 구성 및 형식에 관한 내용은 제 시하지 않고 있으며, 일반적으로 공간프로그램에 포함되는 소요 공간 목록, 크기, 관계에 대한 평가 항목을 언급하고 있다. 따라서 본 연구는 1 차적으로 각 사례별 유효성 평가 항목을 도출하고 이를 앞서 이론적 고찰에서 언급한 공간 프로그램 구성 방식과 비교하였다. 유효성 평가의 해외 사 례의 내용은 다음 [Table 7]과 같다.

[Table 7] Validation Contents

\begin{tabular}{l|c|c|c}
\hline \multicolumn{1}{c|}{ Contents } & GSA & SP & CRC \\
\hline - Whether to match a list of requirements space. & 0 & 0 & 0 \\
\hline - Whether to match of requirements space area. & 0 & 0 & 0 \\
\hline - Whether to match of defined space name. & 0 & 0 & 0 \\
\hline $\begin{array}{l}\text { - Whether to match of Floor area and Gross } \\
\text { area. }\end{array}$ & $\mathrm{n} / \mathrm{a}$ & $\mathrm{O}$ & $\mathrm{n} / \mathrm{a}$ \\
\hline - Accuracy of the measurement of the area. & $\mathrm{n} / \mathrm{a}$ & $\mathrm{O}$ & $\mathrm{n} / \mathrm{a}$ \\
\hline - Area ratio according to the type of building. & $\mathrm{O}$ & $\mathrm{O}$ & $\mathrm{n} / \mathrm{a}$ \\
\hline $\begin{array}{l}\text { - Check of requirements on the spatial } \\
\text { relationship. }\end{array}$ & $\mathrm{n} / \mathrm{a}$ & $\mathrm{n} / \mathrm{a}$ & $\mathrm{O}$ \\
\hline
\end{tabular}

사례 모두 요구 공간 즉, 공간의 목록, 면적에 대한 반영 여부를 검토하고 있으나 평가 결과에 대한 점수 또는 지표 를 사용하고 있지 않았다. $\mathrm{SP}$ 의 경우 면적의 일치 여부를 비중 있게 다루고 GSA의 경우 면적 비율을 통해 보다 세밀 히 평가 하고 있다. $\mathrm{CRC}$ 의 경우 공간 관계에 대한 피난 동 선, 접근성 등의 평가를 포함하고 있다. 특히 유효성 평가 항목 중 공간 명칭에 관한 즉, 설계(BIM 모델)에 사용된 공 간 이름, 유형, 코드에 대해서도 정의된 용어의 일치 여부 를 평가 하고 있다. 이는 공간 프로그램과 설계 안의 내용과 비교 용이하기 위한 방안일 수 있지만 이보단 공간 정보의 일관성을 유지 시켜 참여자간의 의사소통과 정확한 평가를 위한 것으로 판단된다. 즉, 공간 정보의 데이터베이스화를 고려하여 추후 유사 프로젝트 진행시 경험적 정보로서 활 용되기 위한 방안으로 해석된다. 이를 위해 공간의 유형, 코 드 등을 정의하고 제시하고 있다.

이러한 공간 명칭의 정의는 2 가지 방법으로 나타나고 있 


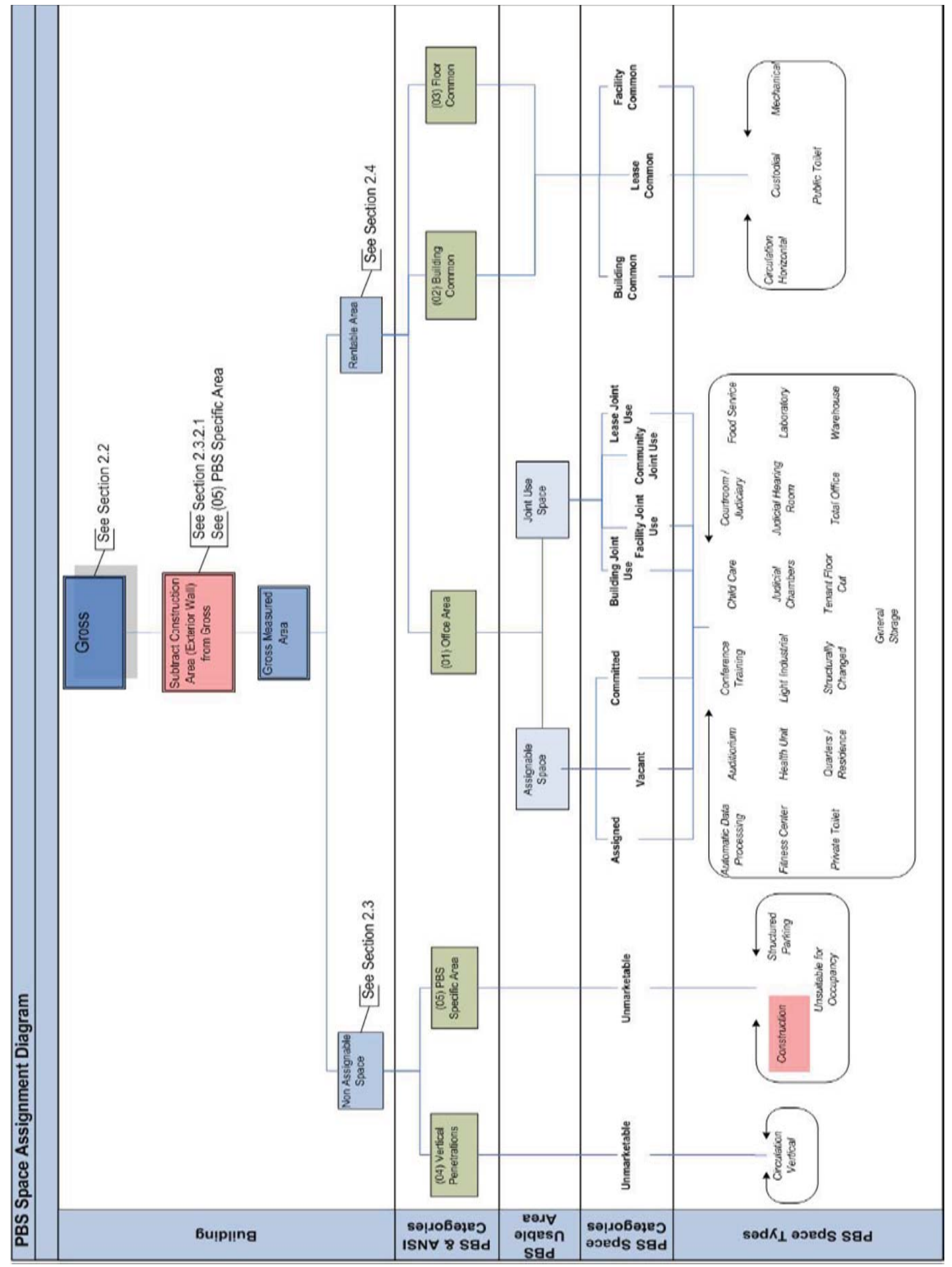

[Figure 6] PBS Space Assignment concept diagram 
다. GSA의 경우 공간의 이름 및 유형에 대해서 구체적으로 제시하고 이에 대한 평가도 비중 있게 다루고 있다. $\mathrm{SP}$ 의 경우 프로젝트 시 참여자간의 협의에 따라 결정하도록 하 고 있으며, $\mathrm{CRC}$ 의 경우 보다 포괄적으로 사용자가 직접 정 할 수 있도록 하고 있다. 이러한 현상은 GSA의 경우 임대사 업을 위한 공간관리 체계를 지속적으로 유지함으로서 보다 효율적인 관리 및 건물의 성능에 관한 연구가 진행되어 나 타난 결과로 미국 GSA의 특수한 경우로 볼 수 있다.

본 연구에서는 사례의 공간 프로그램에 관하여 평가 현 황을 파악하기 위하여 유효성 평가의 세부 내용을 토대로 앞서 이론적 고찰에서 도출된 공간 프로그램의 구성의 내 용을 바탕으로 정리하여 살펴보고자하였다.

[Table 8] Results of Case study on Spatial Program Validation

\begin{tabular}{|c|c|c|}
\hline \multicolumn{2}{|c|}{ Divide } & Contents \\
\hline \multirow{9}{*}{$\begin{array}{l}\text { Space } \\
\text { Requirements }\end{array}$} & \multirow{7}{*}{$\begin{array}{l}\text { Define Space } \\
\text { Information }\end{array}$} & - Defined Space name \\
\hline & & - Defined Space Type \\
\hline & & - Defined Space Area \\
\hline & & - Defined Space Hight \\
\hline & & - Defined Space Unique ID \\
\hline & & - Defined Space Floor \\
\hline & & - Defined user name of Space \\
\hline & $\begin{array}{l}\text { List of } \\
\text { required } \\
\text { space }\end{array}$ & $\begin{array}{l}\text { - Whether to match a list of } \\
\text { requirements space. }\end{array}$ \\
\hline & $\begin{array}{l}\text { Required } \\
\text { space area }\end{array}$ & $\begin{array}{l}\text {-Whether to match of requirements } \\
\text { space area. }\end{array}$ \\
\hline $\begin{array}{l}\text { Specific } \\
\text { facility space } \\
\text { requirements }\end{array}$ & Equipment list & $\mathrm{N} / \mathrm{A}$ \\
\hline \multirow{4}{*}{$\begin{array}{l}\text { Requirements } \\
\text { of the spatial } \\
\text { relationships }\end{array}$} & $\begin{array}{l}\text { Organization } \\
\text { chart }\end{array}$ & - Defined Zone \\
\hline & Work Flow & - Define Zone \\
\hline & \multirow{2}{*}{ Relation Matrix } & - Accessibility \\
\hline & & - Evacuation Route \\
\hline
\end{tabular}

그 결과 공간 별 시설 요구사항을 제외한 공간 요구사항 과 공간 관계 요구사항에 대한 평가가 이루어지고 있으며, $\mathrm{BIM}$ 을 활용하여 구축된 하나의 $\mathrm{BIM}$ 모델의 정보로부터 평 가를 실시하고 있다. 다만, 아직까진 공간 내의 장비에 관한 공간별 시설 요구사항의 내용은 언급하고 있지 않지만 이 는 일반적으로 프로젝트의 특수한 경우를 제외한 공간 시 설에 대한 장비는 계획 설계단계 이후에 주로 결정되므로 조사된 사례의 경우 계획 설계 단계에 초점을 둔 결과로 판 단된다.

\subsection{Efficiency Evaluation}

분석된 사례의 경우 공간 프로그램의 유효성 평가를 위 해 공간 성능 관련 기준 및 지침 등의 활용을 언급하고 있
다. SP와 CRC의 경우 $\mathrm{BIM}$ 을 활용한 공간 프로그램 유효성 평가의 활용 방안에 대해 포괄적으로 접근하고 있어 적용 가능한 지침의 예시정도로만 언급 하고 있으며 GSA의 경우 오피스 건물에 대한 성능 기준을 마련하고 공간프로그램 유효성 평가에 대한 가이드와 성능 지침을 제시하고 있다.

즉, 건축 설계 과정에서의 공간 유효성 평가는 단순히 제 공된 프로그램을 기준으로 BIM 모델에 반영된 설계의 내용 을 단순 비교로서의 내용 보다는 건물 성능의 연구와 관련 된 빌딩 코드 및 기준(building code \& standard)등에 대 한 설계 안의 평가라 볼 수 있다. 이는 프로그램 자체가 건 물의 성능에 관한 분석을 통해 도출된 설계 성능 목표로 서 의미도 있으나 프로그램 안에는 설계자가 이해할 수 있 는 건물 성능 기준에 대한 내용이 포함 되어야 함을 의미하 는 것이다. 사례 별 제시한 성능 기준에 관한 지침은 다음과 [Table 9]와 같다.

[Table 9] Case Status guidance on performance standards

\begin{tabular}{l|l}
\hline \multicolumn{1}{c|}{ Case } & \multicolumn{1}{c}{ Standards } \\
\hline \multirow{3}{*}{ GSA(USA) } & $\cdot$ PBS Business Assignment Guide(2009. 5) \\
\cline { 2 - 2 } & $\begin{array}{l}\text { - ANSI/BOMA Standard Method for Measuring } \\
\text { Floor Area in Office Building (ANSI/BOMA Z } \\
65.1-\text { 1996) }\end{array}$ \\
\hline CRC(Australia) & $\cdot$ NSW BASIX(Building Sustainability Index) \\
\hline SP(Finland) & $\cdot$ N/A \\
\hline
\end{tabular}

GSA의 경우 오피스 건축물에 관한 Public Building Service(이하 PBS)에서 PBS Business Assignment Guide를 제공하고 있으며 이는 GSA의 Spatial Data Management( 이하 $\mathrm{SDM}$ )의 공간 관리 시스템을 활용하기 위한 공간 정 보의 일관성과 정확성을 위한 공간 이름 및 유형, 면적 산정 기준 등의 담고 있다.

또한 미국 국가표준 American National Standard Institute(이하 ANSI)의 Building Owners and Managers Association International(이하 BOMA)의 ${ }^{5)}$ 기준을 기초로 하 고 있다. $\mathrm{BOMA}$ 는 오피스 건축물을 대상으로 경제성 (면적 효율성)에 비중을 두고 있다. 세부 내용은 다음 [Table 10] 과 같다.

호주 $\mathrm{CRC}$ 의 제시된 기준은 적용 가능 기준으로서 언급 하고 있으며, 호주 뉴사우스 웨일즈(New South Wales) 주 의 지속 가능성 건물의 요구조건(Building Sustainability

5) BOMA International은 1915년 오피스건물 면적산출 기준 Standard Method of Floor Measurement for Office Building을 개발하여 1955년 미국 국가 표준(ANSI)으로 인정 되었다. 1996년 6월에 현재 버전인 "Standard Method for Measuring Floor Area in Office Buildings (ANSI/BOMA Z65.1-1996)"을 출판 하였으 며, 오피스빌딩 분야에서 미국 국가 표준을 설정 하는데 주요한 역 할을 수행하고 있다. 


\begin{tabular}{|c|c|}
\hline Items & Content \\
\hline $\begin{array}{l}\text { MAJOR VERTICAL } \\
\text { PENETRATIONS }\end{array}$ & $\begin{array}{l}\text { Stairs, elevator shafts, flues, pipe shafts, vertical ducts, and the like, and their enclosing walls. Atria, light } \\
\text { wells and similar penetrations above the finished floor are included in this definition. Not included, however, } \\
\text { are vertical penetrations built for the private use of a tenant occupying OFFICE AREAS on more than one } \\
\text { floor. Structural columns, openings for vertical electric cable or telephone distribution, and openings for } \\
\text { plumbing lines are not considered to be MAJOR VERTICAL PENETRATIONS. }\end{array}$ \\
\hline FLOOR RENTABLE AREA & $\begin{array}{l}\text { The result of subtracting from the GROSS MEASURED AREA of a floor the MAJOR VERTICAL } \\
\text { PENETRATIONS on that same floor. It is generally fixed for the life of the building and is rarely affected by } \\
\text { changes in corridor size or configuration. }\end{array}$ \\
\hline USABLE AREA & $\begin{array}{l}\text { The measured area of an OFFICE AREA, STORE AREA, or BUILDING COMMON AREA on a floor. The total } \\
\text { of all the USABLE AREAs for a floor shall equal FLOOR USABLE AREA of that same floor. }\end{array}$ \\
\hline OFFICE AREA & $\begin{array}{l}\text { The area where a tenant normally houses personnel and/or furniture, for which a measurement is to be } \\
\text { computed. }\end{array}$ \\
\hline FLOOR USABLE AREA & $\begin{array}{l}\text { The sum of USABLE AREAS of OFFICE AREAS, STORE AREAS and BUILDING COMMON AREAS of a floor. } \\
\text { The amount of FLOOR USABLE AREA can vary over the life of a building as corridors expand and contract } \\
\text { and as floors are remodeled. }\end{array}$ \\
\hline FLOOR COMMON AREA & $\begin{array}{l}\text { The areas on a floor such as washrooms, janitorial closets, electrical rooms, telephone rooms, mechanical } \\
\text { rooms, elevator lobbies, and public corridors which are available primarily for the use of tenants on that } \\
\text { floor. }\end{array}$ \\
\hline BASIC RENTABLE AREA & $\begin{array}{l}\text { The OFFICE AREA, STORE AREA or BUILDING COMMON AREA shall mean the USABLE AREA of that } \\
\text { OFFICE AREA, STORE AREA or BUILDING COMMON AREA and its share of the FLOOR COMMON AREAS } \\
\text { on that floor. BASIC RENTABLE AREA is determined by multiplying the USABLE AREA of that OFFICE AREA, } \\
\text { STORE AREA or BUILDING COMMON AREA by the FLOOR R/U RATIO. The total BASIC RENTABLE AREA } \\
\text { of a tenant occupying more than one floor shall be the sum of its BASIC RENTABLE AREAS on each floor. } \\
\text { The total of all BASIC RENTABLE AREAS on a floor shall equal the FLOOR RENTABLE AREA of that same } \\
\text { floor. }\end{array}$ \\
\hline BUILDING RENTABLE AREA & The sum of all the FLOOR RENTABLE AREAS. \\
\hline BUILDING R/U Ratio & The conversion factor that distributes the BUILDING COMMON AREA of a building. \\
\hline RENTABLE AREA & $\begin{array}{l}\text { The USABLE AREA of an OFFICE AREA or STORE AREA with its associated share of FLOOR COMMON } \\
\text { AREAS and BUILDING COMMON AREAS. RENTABLE AREA is determined by multiplying the USABLE AREA } \\
\text { of an OFFICE AREA or STORE AREA by the R/U RATIO. The total of all RENTABLE AREAS equals the } \\
\text { BUILDING RENTABLE AREA for the building. }\end{array}$ \\
\hline R/U Ratio & $\begin{array}{l}\text { The conversion factor that, when applied to USABLE AREA, gives the RENTABLE } \\
\text { AREA of the OFFICE AREA or STORE AREA. }\end{array}$ \\
\hline
\end{tabular}

Index, 이하 BASIX)을 제시하고 있으며, 이는 주택과 복합 건물의 물, 열, 에너지 사용량에 대한 절약을 통해 온실 가 스 배출량을 줄이고자 하는 호주의 지속 가능형 건물에 관 한 요구사항이다. 이에 대해 건물 공간 면적으로 기반으로 평가 하고 있다. 결론적으로 공간 성능 기준에 대한 사례의 경우 건축물 유형 및 건물의 성능 기준에 대한 비교 분석은 할 수 없으나 공간 프로그램 유효성 평가는 건물의 성능에 대한 기준을 공간 유형별 면적을 기초로 평가하고 있음을 알 수 있다. 또한 이는 건축물의 유형, 가치 등을 고려하여 프로젝트 특성에 따라 적용 기준이 상이 할 수 있는 가능성 이 있다.

$\mathrm{SP}$ 의 경우 다양한 평가 지표 보다는 연면적 대비 순면적 비에 대한 평가와 공간 프로그램 대비 설계안의 반영 비율 에 대하여 검토 하고 있다. 이는 특정 건물 대상 보단 건물 유형의 포괄적으로 접근하는 $\mathrm{SP}$ 의 특성과 공간 프로그램 유효성 평가 절차의 품질관리 관점에 따른 결과로 해석할
수 있다. 또한 GSA의 경우 상업적 성격을 가지는 오피스 건 물을 중심으로 평가하여 공간의 성능에 대해 보다 구체적 으로 제시하고 있다.

$\mathrm{GSA}$ 의 공간 성능에 대하여 건물의 중요 가치는 임대 수 익에 대한 경제성 부분에 초점으로 두고 있다. 따라서 건축 물의 효율성 평가 항목은 건축물의 임대 면적에 대하여 파 악 할 수 있는 면적 유형과 지표로서 구성되어 있으며, 측정 된 면적은 층별 면적, 공용면적, 임대면적, 오피스 면적 등 에 대하여 분석하고 건물 R/U 비율 및 R/U 비율과 같은 지 표를 활용하여 건물의 성능을 쉽게 파악할 수 있도록 하고 있다. 이러한 비율은 건물의 공용면적, 사용 가능면적 등을 산출 할 수 있는 비율로서 활용 된다. 즉, 면적 효율성의 경 우 공간의 성능을 파악하기 위한 지표로서 그 가치가 있지 만 새로운 프로젝트의 초기단계에 건물 규모를 산정하기 위한 지표로서 활용 될 수 있다. 
[Table 11] Space performance assessment items

\begin{tabular}{l|l}
\hline \multicolumn{2}{c}{ Space efficiency assessments (ex.) } \\
\hline \multirow{3}{*}{ Space area } & $\begin{array}{l}\text { Whether the floor area and the total area of } \\
\text { the match, Net area ratio, Public area ratio, } \\
\text { Space program reflects the contrast ratio, } \\
\text { Building R / U ratio }\end{array}$ \\
\hline Space relationships & Accessibility, Evacuation Route \\
\hline
\end{tabular}

면적의 효율성 평가는 공간의 규모에 대한 성능을 평가 하고자 한다면 공간 관계의 요구사항은 각 공간간의 위치 에 따른 성능 평가로서 이는 조직의 구조, 업무의 흐름, 이 동 경로 거리, 각 실의 접근성 등을 파악할 수 있는 평가로 서 공간의 배치 성능에 대한 종합적이 성능 평가를 의미 한 다. 조사된 사례의 경우 공간 관계에 대한 효율성 평가는 법 규와 관련된 피난 동선, 공간의 접근성 등에 대한 평가와 프 로젝트의 특성에 따라 요구되는 공간의 관계에 대해 평가 하고 있다.

공간의 성능에 대하여 사례의 경우 대표적으로 면적 효 율성을 중심으로 평가하고 있는 것으로 볼 수 있다. 면적 의 효율성은 건물의 성능을 전체적으로 파악할 수 있는 평 가 항목이다. 이러한 면적의 효율성은 여러 공간의 배분 면 적 비율로서 건물에 대한 공간 유형별 성능을 평가가 가능 하지만 개별 공간의 성능을 파악하기에는 어려움이 존재한 다. 물론 공간 프로그램 요구 공간 목록 및 크기에 관한 내 용은 각 개별 공간의 성능을 반영한 결과이지만 이는 참여 자간의 개별 공간의 성능에 관한 근거와 목적의 공유가 현 실적으로 어렵다. 따라서 각 개별 공간의 성능에 관한 방안 과 경제성 뿐 만 아니라 사회적, 심리적 다양한 각도에서 평 가할 수 있는 방안이 필요할 것으로 사료된다. 병원건축의 경우 병원의 성능평가는 매우 중요한 항목으로 평가될 수 있다. 따라서 병원의 특수성을 반영한 성능평가지표를 개발 하고 이에 대한 해석을 명확히 하여 기능 중심적인 병원의 목적에 부합하는 연구가 지속적으로 진행되어야 될 필요가 있다.

공간 성능은 공용비, 순면적비, 건물 임대 가능 비율 등 의 면적 효율에 대해서 $\mathrm{CRC}$ 를 제외한 $\mathrm{GSA}$ 와 $\mathrm{SP}$ 에서만 언 급하고 있다. 특히 GSA의 경우 매우 구체적으로 제시하고 있다. 공간 면적을 기반으로 공간의 크기에 관한 평가와 공 간 배치와 관련된 공간 관계에 대한 평가가 이루어지고 있 다. [Table 11] 같이 공간성능에 관한 평가는 공용 면적비, 순면적비, 층별 면적과 총면적의 비교 등의 면적 효율성을 평가하고 있으며 공간 관계에 대한 평가 사항으로는 접근 성, 피난 동선 등의 평가가 이루어지고 있다.

\section{Conclusion}

공간 프로그램 유효성 평가에 대한 해외 사례를 조사 분
석하여 병원건축을 공간 유효성 평가 시 고려하여야 할 사 항들을 정리하여 다음과 같이 결론을 얻을 수 있었다.

1) 공간 프로그램 유효성평가는 다음과 같이 4단계의 평 가 단계로 진행된다.

1 단계 : 기준설정단계(건물요소와 공간에 대한 기준 설 정 등)

2단계 : BIM 모델의 정확성 평가 단계

3단계 : 공간 유효성 평가 단계

4단계 : 성능 평가 단계

2) 기준설정단계에서 건물요소에 대한 기준은 일반 건축 물의 건축요소에 대한 기준을 따라야 할 것으로 생각 되며, 이에 대한 기준이 조속히 국내에서도 제공되어 야 할 과제라고 사료된다.

3) 병원건축에서는 면적산정기준을 국제적인 기준인 $\mathrm{UIA}$ 를 따라 일반건축물의 면적산정기준 차용하여 산 정하는 것이 타당한 것으로 판단된다. 다만, 설계프로 세스 단계별 면적산정방법의 설계효율성을 반영하는 방안을 적용하는 것을 제안한다. 병원건축의 공간 정 의와 체계는 미국 GSA의 경우와 같이 공간 즉 단위 실 들의 명칭과 그에 따른 속성(기능 및 유형 등)을 정의 하여야 하며, 각 단위 실들의 체계를 구축하여야 할 것 으로 판단된다. 기존 연구에서 병원의 부문구성을 체 계화하여 기준을 설정하여야 할 필요가 있다.

4) 병원건축의 경우 일반건축물 보다 다양한 실이 존재 하고 실들 간의 복잡한 관계로 이루어져 있다. 따라서 최적의 설계안을 도출하기 위해 계획된 공간의 적정 규모, 배치, 공간간의 관계를 종합적으로 판단할 수 있 는 병원의 성능평가가 매우 중요하다. 따라서 병원의 특수성을 반영한 성능평가지표를 개발하고 이에 대한 해석을 명확히 하여 기능 중심적인 병원의 목적에 부 합하는 연구가 지속적으로 진행되어야 될 필요가 있 다.

Acknowledgements: This work was supported by Academic Research of Chungwoon University Grant funded in 2012

\section{References}

1. Ministry of Construction \& Transportation, Korea Institute of Construction \& Transportation Technology Evaluation and Planning, Exhibition and publicity of research performance in future growth engine, 2007, p.4

2. ANSI/BOMA, BOMA International, Standard Method for Measuring Floor Area in Office Buildings, 1996, pp.5-26

3. Choi, Jong-Chun; Kim, Khil-chea, A study on the preconditions of BlM based design process, Korean Digital 
Architecture-Interior Association, 2008.08

4. General Services Administration, BIM Guide Series 02 Spatial Program Validation” 2007, pp.2-11

5. General Services Administration Public Building Service, National Business Space Assignment Policy, 2009, pp.1-27

6. Jun, Young-il, Architectural Design Theory, kimoondang, 1997, pp.286-388

7. Kim, Kwang-Moon, Space programming methods, kimoondang, 1988, p.33

8. Park, Young-Sup, The Development of the Spatial Layout Evaluation Model (SLEM) in Terms of Space Program Validation, A doctoral dissertation Seoul University, 2009, pp. 29

9. Senate Properties(Finland), BIM Requirements 2007 Vol 6, 2007, pp.20-35

10. Seong, Joon-ho; Kim, Khil-chea, A Study on Spatial Program Validation of General Service Administration, Architectural Insitute of Korea, 2009.10

접수 : 2013년 3월 29일 1차 심사 완료 : 2013년 4월 19일 게재확정일자 : 2013년 4월 19일 3 인 익명 심사 필 